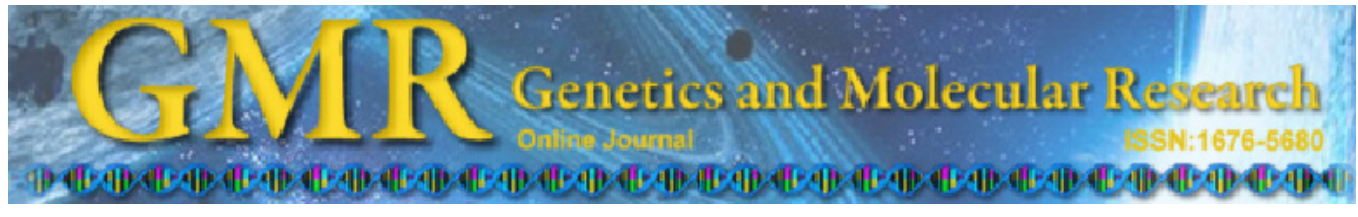

\title{
Gene expression study related with the intrinsic pathway of apoptosis in bladder cancer by real-time PCR technique
}

\author{
D.F. Barione ${ }^{1}$, F.S.N. Lizarte ${ }^{1}$, P.C. Novais ${ }^{1}$, C.A.M. de Carvalho ${ }^{1}$, \\ F.C.B. Valeri' ${ }^{1}$, F.M. Peria ${ }^{3}$, H.F. de Oliveira ${ }^{3}$, D.L. Zanette ${ }^{2}$, \\ W.A. Silva Jr. ${ }^{2}$, A.J. Cologna ${ }^{1}$, R.B. Reis ${ }^{1}$, S. Tucci Jr. ${ }^{1}$, \\ A.C.P. Martins ${ }^{1}$, D.P.C. Tirapelli ${ }^{1}$ and L.F. Tirapelli ${ }^{1}$ \\ ${ }^{1}$ Departamento de Cirurgia e Anatomia, \\ Faculdade de Medicina de Ribeirão Preto, Universidade de São Paulo, \\ Ribeirão Preto, SP, Brasil \\ ${ }^{2}$ Departamento de Genética, Faculdade de Medicina de Ribeirão Preto, \\ Universidade de São Paulo, Ribeirão Preto, SP, Brasil \\ ${ }^{3}$ Departamento de Clínica Médica, Faculdade de Medicina de Ribeirão Preto, \\ Universidade de São Paulo, Ribeirão Preto, SP, Brasil
}

Corresponding author: L.F. Tirapelli

E-mail: tirapeli@fmrp.usp.br

Genet. Mol. Res. 12 (2): 878-886 (2013)

Received February 6, 2012

Accepted December 20, 2012

Published April 2, 2013

DOI http://dx.doi.org/10.4238/2013.April.2.4

\begin{abstract}
We examined the expression of anti-apoptotic genes (XIAP and Bcl-2) and apoptotic genes (cytochrome c, caspase-9, Apaf$1)$ in tissue samples of patients with superficial bladder cancer. Thirtytwo bladder cancer tissue samples ( 8 papillary urothelial neoplasm of low malignant potential, 10 low-grade, and 14 high-grade) and 8 normal bladder tissue samples from necropsy were used for the study of gene expression by real-time PCR analysis. Analysis of the expression of apoptotic gene constituents of an apoptosome demonstrated an increase in Apaf-1 expression in the three tumor grades when compared with the control ( $\mathrm{P}<0.01, \mathrm{P}<0.05$, and $\mathrm{P}<0.01)$, low expression of caspase- 9 in all groups $(\mathrm{P}<0.05)$, and an increase in cytochrome $c$ expression in
\end{abstract}


all tumor grades in relation to the control, although without statistically significant difference. The expression of anti-apoptotic genes revealed an increase in XIAP expression in all tumor grades in relation to the control, although without statistically significant difference, and low expression of $B c l-2$ in all tumor grades and the control $(\mathrm{P}<0.05)$. The results proved that there is low evidence of apoptotic activity by the intrinsic pathway, demonstrated by the low expression of caspase- 9 and considerable increase in XIAP expression, which may render these genes potential therapeutic targets in bladder cancer treatment.

Key words: Bladder cancer; Apoptosis; Gene expression; RT-PCR

\section{INTRODUCTION}

Bladder cancer is the fifth most common neoplasm diagnosed in the Occident, being diagnosed approximately 336,000 new cases annually and killing 132,000 patients around the world (Parkin et al., 2001a,b; Pashos et al., 2002). After a quarter century of rapid advances, cancer research has generated a rich and complex body of knowledge, revealing cancer to be a disease involving dynamic changes in the genome (Hanahan and Weinberg, 2000).

The vast catalog of cancer cell genotypes is a manifestation of six essential alterations in cell physiology that collectively dictate malignant growth: self-sufficiency in growth signals, insensitivity to growth-inhibitory (antigrowth) signals, limitless replicative potential, sustained angiogenesis, tissue invasion, and evasion of programmed cell death (apoptosis) (Hanahan and Weinberg, 2000), the last being the focus of this study.

Apoptosis is an evolutionarily conserved, genetically controlled process of programmed cell death used by multicellular organisms to eliminate cells in diverse physiological settings such as development, homeostasis of tissues, and maintenance of integrity of the organism (Raff, 1998; Ameisen, 2002). Two major pathways leading to apoptosis have been delineated: the extrinsic or receptor-mediated pathway, and the intrinsic or mitochondrial pathway. Both pathways involve the activation of a cascade of enzymes called caspases, a family of cysteine proteases that cleave after aspartic acid residues. The extrinsic and intrinsic pathways possess an independent group of initiator caspases (which activates effector caspases), and the pathways converge to utilize the same group of effector caspases that execute the final cell death program (Budihardjo et al., 1999).

The extrinsic pathway is characterized by the activation of cell surface death receptors following binding of their specific ligand (Ashkenazi and Dixit, 1998). The intrinsic pathway can be executed independent of death receptor signaling, also resulting in the activation of effector caspases. In a now well-characterized process, mitochondrial damage results in the leakage of cytochrome $c$ into the cytoplasm. Subsequently, cytochrome c complexes with the cytoplasmic protein Apaf-1, which then oligomerizes and binds to procaspase-9, resulting in the formation of a multimeric complex called the apoptosome. This brings procaspase- 9 molecules into close proximity with each other, allowing enzymatic self-activation. Caspase-9 is then able to cleave and activate the downstream effector caspases-3, 6, and 7 (Liu et al., 1996; Li et al., 1997; Zou et al., 1997).

Understanding the molecular mechanism and regulation of apoptotic function in dis- 
ease states is important for understanding such states and how they might be best treated. Thus, our objective was to analyze the genes that constitute the apoptosome (Apaf-1, cytochrome c, and caspase-9) and anti-apoptotic genes (Bcl-2 and XIAP, known to regulate the intrinsic pathway) in human samples of superficial bladder cancer.

\section{PATIENTS AND METHODS}

The Ethics Committee in Research at Hospital das Clínicas da Faculdade de Medicina de Ribeirão Preto (HCFMRP) approved the protocol and all patients permitted the use of resected material from their surgery by signing an informed consent form. Thirty-two samples of bladder superficial tumors were stored in liquid nitrogen immediately after their resection. They were graded as papillary urothelial neoplasm of low malignant potential (PUNLMP): 8 samples, low-grade papillary urothelial carcinoma: 10 samples, and highgrade papillary urothelial carcinoma: 14 samples. Eight samples from necropsy of patients who did not have urogenital problems in life were used as control.

The RNA was extracted using the Trizol reagent (Invitrogen, Carlsbad, CA, USA) as recommended by the manufacturer, and the quality of the samples was assessed by $1 \%$ agarose gel electrophoresis. We used the High Capacity cDNA Reverse Transcription Kit (Applied Biosystems, Foster City, CA, USA) to synthesize cDNA, and TaqMan Master Mix (Applied Biosystems) and TaqMan Assay-on-Demand (Applied Biosystems) according to manufacturer instructions to perform the real-time PCR. The Assays-on-Demand were: caspase-9 (Assay ID Hs00154260_m1), cytochrome c (Assay ID Hs01588973_m1), Apaf-1 (Assay ID Hs00559441_m1), XIAP (Assay ID 00236913_m1), Bcl-2 (Assay ID 00608023_m1), and SDHA (Assay ID Hs00188166_m1), the last being used as the endogenous reference gene (Ohl et al., 2006). The median $\Delta$ Cts of control samples was used as the calibrator.

Analysis was conducted using one-way ANOVA and the Tukey post-test with GraphPad Prism version 4.0 for Windows (GraphPad Software, San Diego, CA, USA). Values of $\mathrm{P}<0.05$ were considered to be statistically significant.

\section{RESULTS}

The gene expression analysis presented normal distribution. The Apaf-1 gene expression (Figure 1) in all tumor groups exhibited increased expression when compared with the control. A statistically significant difference was observed between PUNLMP and control, PUNLMP and low-grade tumor, and PUNLMP and high-grade tumor $(\mathrm{P}<0.0001$, one-way ANOVA; $\mathrm{P}<0.01, \mathrm{P}<0.05$, and $\mathrm{P}<0.01$, respectively, Tukey post-test).

Caspase- 9 gene expression is illustrated in Figure 2. We observed low expression of this gene in all groups of samples. A statistically significant difference was observed in PUNLMP and low-grade tumor ( $\mathrm{P}=0.0007$, one-way ANOVA; $\mathrm{P}<0.05$, Tukey post-test).

Although the cytochrome $c$ gene in all groups of samples presented increased expression (Figure 3), there was no statistically significant difference ( $\mathrm{P}=0.207$, one-way ANOVA).

A statistically significant difference was observed between $B c l-2$ expression in PUNLMP and high-grade tumors ( $\mathrm{P}=0.0007$, one-way ANOVA; $\mathrm{P}<0.05$, Tukey post-test $)$ (Figure 4). However, all groups studied presented low expression of this gene. 


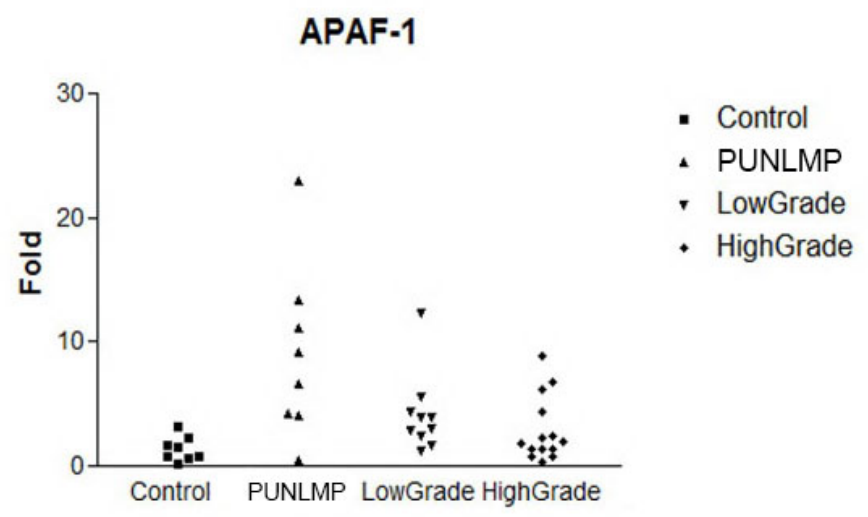

Figure 1. Median values ( \pm standard deviation) of APAF-1 expression in superficial bladder tumors and control samples ( $\mathrm{P}<0.0001$, one-way ANOVA; C x GI, P $<0.01$; GI x GII, P $<0.05$; GI x GIII, $\mathrm{P}<0.01$, Tukey post-test).

\section{Caspase-9}

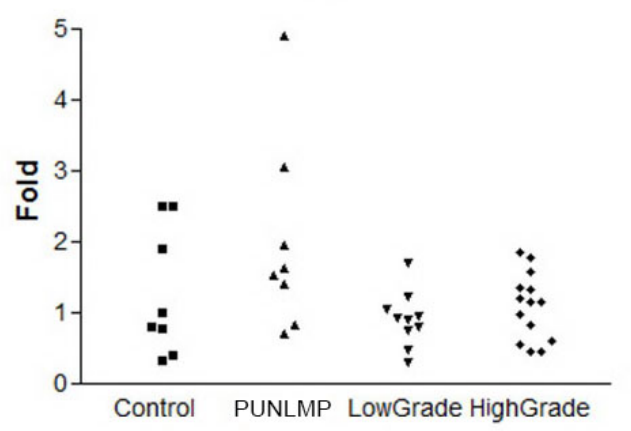

- Control

- PUNLMP

- LowGrade

- HighGrade

Figure 2. Median values ( \pm standard deviation) of caspase-9 expression in superficial bladder tumors and control samples $(\mathrm{P}=0.0007$, one-way ANOVA; GI x GII, $\mathrm{P}<0.05$, Tukey post-test).

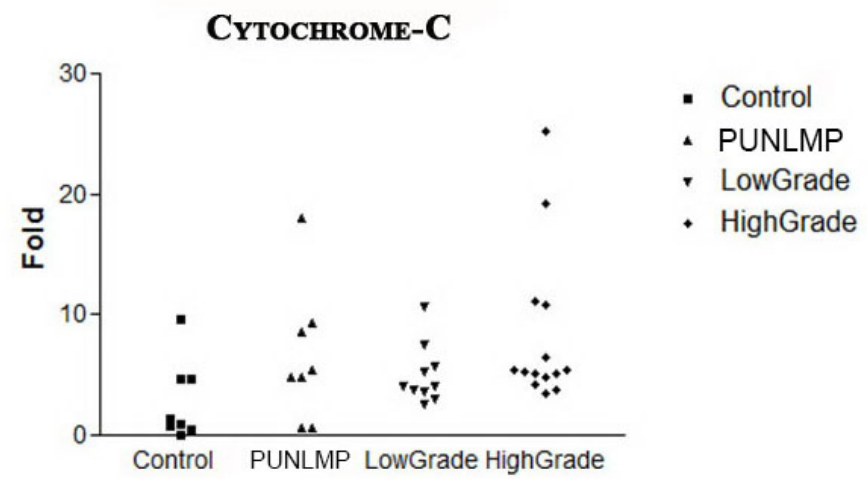

Figure 3. Median values ( \pm standard deviation) of cytochrome-c expression in superficial bladder tumors and control samples $(\mathrm{P}=0.207$, one-way ANOVA). 


\section{$\mathrm{BCl}-2$}

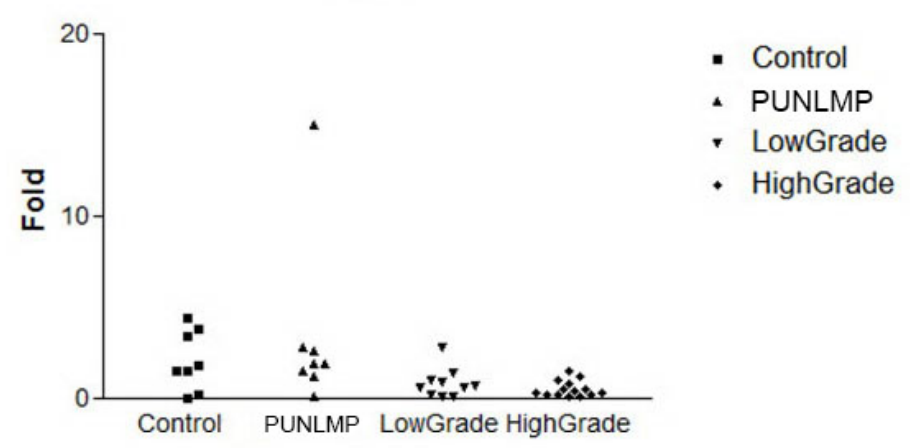

Figure 4. Median values ( \pm standard deviation) of BCL-2 expression in superficial bladder tumors and control samples ( $\mathrm{P}=0.0007$, one-way ANOVA; GI $\mathrm{x}$ GIII, $\mathrm{P}<0.05$, Tukey post-test).

Figure 5 depicts increased expression of XIAP in PUNLMP, low-grade tumor, highgrade tumor, and the control. No statistically significant difference was noted $(\mathrm{P}=0.1616$, one-way ANOVA).

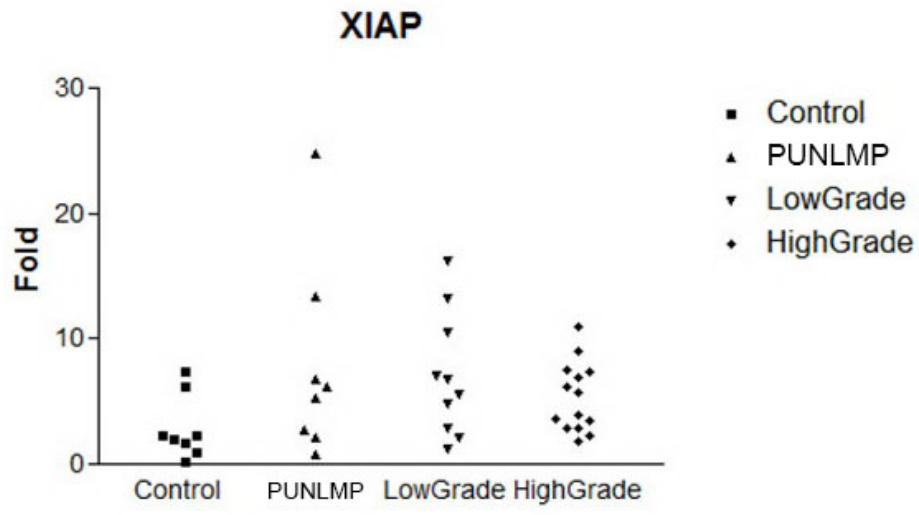

Figure 5. Median values ( \pm standard deviation) of XIAP expression in superficial bladder tumors and control samples $(\mathrm{P}=0.1616$, one-way ANOVA).

\section{DISCUSSION}

Jia et al. (2001) were the first to provide direct evidence suggesting that Apaf-1 protein levels in human leukemic cells vary constitutively. In addition, we demonstrated that a relative deficiency of Apaf-1 in human leukemic cells and tumor cells can result in resistance to apoptosis. Restoration of Apaf-1 protein levels after gene transfection is able to resensitize previously resistant cells to UV light-induced killing. Comparing two different cell lines, Jia et al. (2001) 
observed that the bladder cancer J82 cell line expressed less Apaf-1 protein than the CEM/ $\mathrm{VLB}_{100}$ cell line, and that the sensitivity of J82 cells to UV light-induced apoptosis was considerably lower than that in $\mathrm{CEM} / \mathrm{VLB}_{100}$ cells. The percentage of apoptosis and the activation of caspase-3 and 9- were significantly increased after transfection with the Apaf-1 gene. Moreover, Apaf-1 transfection suppressed colony formation in J82 cells after UV light stimulation.

Christoph et al. (2008) investigated the methylation status of Apaf-1, caspase-8, $D A P K-1$, and $I G F B P-3$ in bladder, kidney, and testicular cancer using real-time methylationspecific PCR to find similarities in methylation frequency and individual levels of methylation with regard to histopathological classification and biological behavior. Methylation frequency was the highest for Apaf-1 (88-100\%) for all the different tumor types. The highest Apaf-1 and $D A P K-1$ levels of methylation were found in the advanced tumor groups. Methylation frequency and methylation levels in normal tissue were significantly lower. Apaf-1 methylation was also frequent in normal testicular tissue, but at lower levels than that in cancerous tissue. Apaf-1 methylation levels in recurrent superficial and muscle-invasive bladder tumors were significantly higher. Methylation levels of Apaf- 1 were also correlated with higher risk for recurrent metastatic disease in non-advanced tumors of the kidney. The methylation profile observed demonstrated a substantial role of the Apaf- 1 gene in tumorigenesis. The different methylation pattern in testicular cancer might represent a different methylotype of this cancer.

The Apaf-1 gene is frequently methylated in bladder cancer, and methylation of this gene is found in more aggressive tumor types. Hinz et al. (2007) studied the EZH2 gene, which controls methylation of various EZH2 target promoters and the methylation of Apaf-1, DAPK1, and IGFBP-3 in transitional cell carcinoma (TCC). The EZH2 expression levels correlated well with the differentiation grade of the tumor specimens $(\mathrm{P}=0.03)$, and the Apaf-1 methylation correlated with tumor stage $(\mathrm{P}=0.0001)$ and grade $(\mathrm{P}=0.004)$. Matched-pair analysis demonstrated a statistically significant correlation between elevated EZH2 mRNA expression and higher methylation levels of Apaf- 1 in superficial $(\mathrm{P}=0.024)$ and well-differentiated $(\mathrm{P}=$ $0.04)$ TCC. Apaf-1 and IGFBP-3 methylation levels were significantly higher $(\mathrm{P}=0.03$ and $\mathrm{P}$ $=0.01$, respectively) in patients with recurrent TCC. The authors concluded that $E Z H 2$ expression and Apaf-1 methylation are related to tumor progression and invasiveness.

Apaf- 1 is necessary to activate caspase- 9 , which activates caspase- $3,-6$, and -7 (Furukawa et al., 2005). Inactivation of Apaf-1 is described in malignant melanomas and malignant hematopoietic disorders (Soengas et al., 2001; Fu et al., 2003). Apaf-1 expression is reactivated following exposure to demethylation reagents, demonstrating the relationship between re-expression and methylation. Fu et al. (2003) restored mRNA and protein expression in leukemic cell lines with altered Apaf-1. Christoph et al. (2008) highlighted the importance of Apaf-1 in tumorigenesis and suggested that the inactivation of this gene by methylation is an early event in bladder cancer progression.

In our study, we noted that the expression of Apaf-1 in PUNLMP was significantly higher in relation to low- and high-grade tumors, and the expression decreased as the histological grade increased. Although there were no statically significant differences between lowand high-grade tumors, low-grade tumors exhibited higher expression than high-grade tumors, demonstrating that the gene expression decreases when the lesion becomes more aggressive. These data are in accordance with those of other studies (Hinz et al., 2007; Christoph et al., 2007,2008 ), which proves that the apoptotic rate can decrease in aggressive lesions, as Apaf-1 is an important factor that controls the intrinsic pathway of apoptosis (Furukawa et al., 2005). 
The role of cytochrome $c$ in apoptosis was first identified in experiments in which the addition of dATP to cytosolic extracts induced caspase activity (Liu et al., 1996). Immunodepletion of cytochrome $c$ in an extract abolished its apoptotic potential, whereas restoration of cytochrome $c$ led to the recovery of apoptotic potential (Ow et al., 2008).

The connection to mitochondria was first observed in a Xenopus egg extract system in which mitochondria were required for spontaneous caspase activation, and cytochrome $c$ was shown to be the main mediator of this effect (Newmeyer et al., 1994; Kluck et al., 1997).

Subsequently, microinjection of cytochrome $c$ into the cytosol of various mammalian cell types was found to induce apoptosis (Li et al., 1997; Zhivotovsky et al., 1998). Conversely, cytochrome c-deficient cells were resistant to various apoptotic stimuli and exhibited decreased activation of caspase-3. Addition of exogenous cytochrome $c$ to the mutant cells abrogated resistance to apoptosis ( $\mathrm{Li}$ et al., 2000).

In our results, we found no statically significant difference for cytochrome $c$ expression. The gene expression in all tumor grades and the control was similar, and high-grade tumors presented a slight increase in relation to the other groups. Nevertheless, we cannot suggest that apoptosis by the intrinsic pathway is increased.

Bcl-2 proteins are described as mitochondrial membrane regulators, and one of their functions is to control cytochrome $c$ release, blocking the intrinsic pathway mechanism (Cory and Adams, 2002). The role of $B c l-2$ expression in cellular tumorigenesis was demonstrated in transgenic rats, in which super-expression developed lymphoid tumors (Strasser et al., 1990). The high expression of $\mathrm{Bcl}-2$ has been noted in many human cancers (hematopoietic, prostate, breast) (Reed et al., 1996).

In bladder cancer, immunoreactivity is significantly higher in poorly differentiated tumors (King et al., 1996). However, Nakanishi et al. (1998) identified high immunoreactivity in patients with low-grade tumors. The role of Bcl-2 in TCC is not very clear (Bilim et al., 1998). Shiina et al. (1996) demonstrated high expression associated with less aggressive tumors, whereas Bilim et al. (1998) reported high expression in aggressive tumors.

Despite a significant difference between $B c l-2$ expression in PUNLMP and highgrade tumors, $B c l-2$ expression in all groups was very low, and expression was the lowest in the high-grade tumors. Therefore, our findings are in accordance with those of previous reports (Shiina et al., 1996; Cristoph et al., 2007). In our results, the expression of $B c l-2$ clarifies the expression profile of cytochrome $\mathrm{c}$. As an anti-apoptotic gene, $\mathrm{Bcl}-2$ expression does not block the higher expression of cytochrome $c$ released from the mitochondria. This connection is more obvious when comparing high-grade tumors in both genes (Figures 3 and 4).

Another group of proteins that control apoptosis are the members of the IAP family (Shi, 2002; Riedl and Shi, 2004). They control the activity of initiator and effector caspases (Deveraux and Reed, 1999). XIAP is a well-characterized member of this family and a powerful inhibitor of apoptosis (Salvesen and Duckett, 2002).

Increased expression of XIAP is reported in many cancers, including bladder cancer, and can produce resistance to chemotherapy drugs (Deveraux et al., 1999; Parton et al., 2002; Bilim et al., 2003). Li et al. (2007) demonstrated that XIAP expression was present in 61 samples, or $4 \%$ of non-invasive bladder tumors. There was no statically significant difference between the expression and grade. However, it was noted that well-differentiated tumors (G1) expressed more XIAP than poorly differentiated ones (G3), which is similar to the findings of another study on different cancer types (Takeuchi et al., 2005). High levels of XIAP in super- 
ficial bladder tumors contribute to decrease of apoptotic activity, providing an advantage in tumorigenesis (Li et al., 2007).

The expression of caspase-9 in our study revealed a significant difference between PUNLMP and high-grade tumors. Nevertheless, gene expression in all groups analyzed was low.

The low caspase- 9 expression can be explained by analyzing XIAP expression, which can inhibit caspase-9. There were no significant differences in XIAP expression, which is in accordance with the findings of $\mathrm{Li}$ et al. (2007).

We suggest that the apoptotic activity by the intrinsic pathway in superficial bladder cancer is very low because caspase- 9 expression prevents apoptosome formation, and consequently, activation of effector caspases. In addition, the high expression of XIAP may inhibit caspase- 9 activity.

However, we cannot assume that apoptosis is absent in these tumors, because this process can occur by the intrinsic pathway, which is regulated by another group of proteins.

\section{ACKNOWLEDGMENTS}

Research supported by FAEPA-HCFMRP (Fundação de Apoio ao Ensino, Pesquisa e Assistência do Hospital das Clínicas, FMRP-USP).

\section{REFERENCES}

Ameisen JC (2002). On the origin, evolution, and nature of programmed cell death: a timeline of four billion years. Cell Death Differ. 9: 367-393.

Ashkenazi A and Dixit VM (1998). Death receptors: signaling and modulation. Science 281: 1305-1308.

Bilim VN, Tomita Y, Kawasaki T, Takeda M, et al. (1998). Variable Bcl-2 phenotype in benign and malignant lesions of urothelium. Cancer Lett. 128: 87-92.

Bilim V, Kasahara T, Hara N, Takahashi K, et al. (2003). Role of XIAP in the malignant phenotype of transitional cell cancer (TCC) and therapeutic activity of XIAP antisense oligonucleotides against multidrug-resistant TCC in vitro. Int. J. Cancer 103: 29-37.

Budihardjo I, Oliver H, Lutter M, Luo X, et al. (1999). Biochemical pathways of caspase activation during apoptosis. Annu. Rev. Cell Dev. Biol. 15: 269-290.

Christoph F, Hinz S, Kempkensteffen C, Weikert S, et al. (2007). A gene expression profile of tumor suppressor genes commonly methylated in bladder cancer. J. Cancer Res. Clin. Oncol. 133: 343-349.

Christoph F, Hinz S, Weikert S, Kempkensteffen C, et al. (2008). Comparative promoter methylation analysis of p53 target genes in urogenital cancers. Urol. Int. 80: 398-404.

Cory S and Adams JM (2002). The Bcl2 family: regulators of the cellular life-or-death switch. Nat. Rev. Cancer 2: 647-656.

Deveraux QL and Reed JC (1999). IAP family proteins - suppressors of apoptosis. Genes Dev. 13: 239-252.

Deveraux QL, Leo E, Stennicke HR, Welsh K, et al. (1999). Cleavage of human inhibitor of apoptosis protein XIAP results in fragments with distinct specificities for caspases. EMBO J. 18: 5242-5251.

Fu WN, Bertoni F, Kelsey SM, McElwaine SM, et al. (2003). Role of DNA methylation in the suppression of Apaf-1 protein in human leukaemia. Oncogene 22: 451-455.

Furukawa Y, Sutheesophon K, Wada T, Nishimura M, et al. (2005). Methylation silencing of the Apaf-1 gene in acute leukemia. Mol. Cancer Res. 3: 325-334.

Hanahan D and Weinberg RA (2000). The hallmarks of cancer. Cell 100: 57-70.

Hinz S, Kempkensteffen C, Weikert S, Schostak M, et al. (2007). EZH2 polycomb transcriptional repressor expression correlates with methylation of the APAF-1 gene in superficial transitional cell carcinoma of the bladder. Tumour. Biol. 28: 151-157.

Jia L, Srinivasula SM, Liu FT, Newland AC, et al. (2001). Apaf-1 protein deficiency confers resistance to cytochrome c-dependent apoptosis in human leukemic cells. Blood 98: 414-421.

King ED, Matteson J, Jacobs SC and Kyprianou N (1996). Incidence of apoptosis, cell proliferation and bcl-2 expression in transitional cell carcinoma of the bladder: association with tumor progression. J. Urol. 155: 316-320. 
Kluck RM, Bossy-Wetzel E, Green DR and Newmeyer DD (1997). The release of cytochrome c from mitochondria: a primary site for Bcl-2 regulation of apoptosis. Science 275: 1132-1136.

Li K, Li Y, Shelton JM, Richardson JA, et al. (2000). Cytochrome c deficiency causes embryonic lethality and attenuates stress-induced apoptosis. Cell 101: 389-399.

Li M, Song T, Yin ZF and Na YQ (2007). XIAP as a prognostic marker of early recurrence of nonmuscular invasive bladder cancer. Chin. Med. J. 120: 469-473.

Li P, Nijhawan D, Budihardjo I, Srinivasula SM, et al. (1997). Cytochrome c and dATP-dependent formation of Apaf-1/ caspase-9 complex initiates an apoptotic protease cascade. Cell 91: 479-489.

Liu X, Kim CN, Yang J, Jemmerson R, et al. (1996). Induction of apoptotic program in cell-free extracts: requirement for dATP and cytochrome c. Cell 86: 147-157.

Nakanishi K, Tominaga S, Kawai T, Torikata C, et al. (1998). Expression of bcl-2 oncoprotein in transitional cell carcinoma of the upper urinary tract. Virchows Arch. 432: 445-450.

Newmeyer DD, Farschon DM and Reed JC (1994). Cell-free apoptosis in Xenopus egg extracts: inhibition by Bcl-2 and requirement for an organelle fraction enriched in mitochondria. Cell 79: 353-364.

Ohl F, Jung M, Radonic A, Sachs M, et al. (2006). Identification and validation of suitable endogenous reference genes for gene expression studies of human bladder cancer. J. Urol. 175: 1915-1920.

Ow YP, Green DR, Hao Z and Mak TW (2008). Cytochrome c: functions beyond respiration. Nat. Rev. Mol. Cell Biol. 9: $532-542$.

Parkin DM, Bray F, Ferlay J and Pisani P (2001a). Estimating the world cancer burden: Globocan 2000. Int. J. Cancer 94: 153-156.

Parkin DM, Bray FI and Devesa SS (2001b). Cancer burden in the year 2000. The global picture. Eur. J. Cancer 37 (Suppl 8): S4-66.

Parton M, Krajewski S, Smith I, Krajewska M, et al. (2002). Coordinate expression of apoptosis-associated proteins in human breast cancer before and during chemotherapy. Clin. Cancer Res. 8: 2100-2108.

Pashos CL, Botteman MF, Laskin BL and Redaelli A (2002). Bladder cancer: epidemiology, diagnosis, and management. Cancer Pract. 10: 311-322.

Raff M (1998). Cell suicide for beginners. Nature 396: 119-122.

Reed JC, Miyashita T, Takayama S, Wang HG, et al. (1996). BCL-2 family proteins: regulators of cell death involved in the pathogenesis of cancer and resistance to therapy. J. Cell Biochem. 60: 23-32.

Riedl SJ and Shi Y (2004). Molecular mechanisms of caspase regulation during apoptosis. Nat. Rev. Mol. Cell Biol. 5: 897-907.

Salvesen GS and Duckett CS (2002). IAP proteins: blocking the road to death's door. Nat. Rev. Mol. Cell Biol. 3: 401-410.

Shi Y (2002). Mechanisms of caspase activation and inhibition during apoptosis. Mol. Cell 9: 459-470.

Shiina H, Igawa M, Urakami S, Honda S, et al. (1996). Immunohistochemical analysis of bcl-2 expression in transitional cell carcinoma of the bladder. J. Clin. Pathol. 49: 395-399.

Soengas MS, Capodieci P, Polsky D, Mora J, et al. (2001). Inactivation of the apoptosis effector Apaf-1 in malignant melanoma. Nature 409: 207-211.

Strasser A, Harris AW, Bath ML and Cory S (1990). Novel primitive lymphoid tumours induced in transgenic mice by cooperation between myc and bcl-2. Nature 348: 331-333.

Takeuchi H, Kim J, Fujimoto A, Umetani N, et al. (2005). X-Linked inhibitor of apoptosis protein expression level in colorectal cancer is regulated by hepatocyte growth factor/C-met pathway via Akt signaling. Clin. Cancer Res. 11: 7621-7628.

Zhivotovsky B, Orrenius S, Brustugun OT and Doskeland SO (1998). Injected cytochrome c induces apoptosis. Nature 391: 449-450.

Zou H, Henzel WJ, Liu X, Lutschg A, et al. (1997). Apaf-1, a human protein homologous to C. elegans CED-4, participates in cytochrome c-dependent activation of caspase-3. Cell 90: 405-413. 